\title{
Digital Gangrene in Disseminated Tuberculosis: A Rare Presentation
}

\author{
Dr Naman Bansal ${ }^{1}$, Dr VK Tyagi ${ }^{2}$ \\ ${ }^{I}$ (Department of Medicine, Subharti Medical College, India) \\ ${ }_{2}^{2}$ (Department of Medicine, Subharti Medical College, India)
}

\begin{abstract}
Peripheral gangrene as a manifestation of tuberculosis is rarely seen. A 35 year old male presented with history of fever, lymphadenitis and bluish discolouration of the ring and little finger of the left hand. A diagnosis of tuberculosis was based on history, favourable radiological and epidemiological features and suggestive laboratory investigations. Patient was started on ATT and improved dramatically. We present a case of such rare presentation.
\end{abstract}

Keywords: ATT, Gangrene, Medium-vessel, Tuberculosis, Vasculitis,

\section{Introduction}

India is the country with the highest burden of TB. The World Health Organization (WHO) gives an estimated incidence figure of 2.5 million cases of TB for India out of a global incidence of 9 million ${ }^{(1)}$. It is estimated that about $40 \%$ of the Indian population is infected with latent TB bacteria. Tuberculosis has a varied clinical spectrum and can have various manifestations. Digital gangrene is rarely seen in tuberculosis. ${ }^{(2,3)} \mathrm{We}$ present a patient of disseminated tuberculosis which induced medium vessel vasculitis and caused digital gangrene.

\section{Case Report}

A 35 year old male presented with history of fever, lymphadenitis and bluish discoloration of the ring and little finger of the left hand. One year ago, the patient experienced low grade fever associated with evening rise, night sweats and cough with expectoration. Tuberculin skin test was positive and the Chest X-Ray revealed fibro-cavitatory lesion in the left upper zone, which suggested possible pulmonary tuberculosis. The patient had chronic lower back pain and tenderness in L4 and L5 lumbar vertebra. MRI was done and a diagnosis of pott's spine was made. The patient also had minimal pleural effusion and mild ascites. An ascitic tap was done but it was unsuccessful (dry). The patient was started on standard ATT with isoniazide, rifampicin, pyrazinamide and ethambutol. Two months later, the symptoms resolved but the patient stopped taking the medication on his own.

A month ago, the patient had severe pain and tenderness in the fingers of left hand. The symptoms gradually worsened and the fingers turned blue. When the patient presented to us, two fingers had turned blue and the third had severe pain and tenderness. There were no purpuric or erythematous or nodular skin lesions or bleeding from any site of the body. The patient was a non smoker and belonged to low socio-economic status according to modified Kuppuswamy scale. The patient was admitted for further evaluation.

The patient's general condition was fair. His height was $173 \mathrm{~cm}$ and weight was $51 \mathrm{~kg}$ (BMI - 17). His blood pressure in the right upper limb was 130/86 mmHg, in the right lower limb was 132/84 $\mathrm{mmHg}$, in the left upper arm was $64 / 30 \mathrm{mmHg}$ and in the left lower limb was $130 / 80 \mathrm{mmHg}$. The pulsations of left radial, left ulnar and left brachial artery were weak. A portable handheld Vascular Doppler was used to confirm the sluggish flow. The left hand had lower temperature compared to right. The patient also had enlarged left axillary lymph nodes.

The patient had an RBS of $86 \mathrm{mg} / \mathrm{dl}$, Erythrocytic Sedimentation Rate (ESR) of $90 \mathrm{~mm} / \mathrm{s}^{\mathrm{st}}$ hour, CRP of $31.1 \mathrm{mg} / \mathrm{dl}$ against a normal value of $10 \mathrm{mg} / \mathrm{dl}$. RA factor was negative. Complete Blood count, Liver function tests, Kidney function tests, S.Electrolytes and Lipid Profile were normal. Urine R/M showed 3+ albumin but no RBC were seen. His coagulation profile was normal. Mantoux test with 5 tuberculin unit purified protein derivative was strongly positive with an induration of $15 \mathrm{~mm}$. Serological markers for Hepatitis B and C and human immunodeficiency virus (HIV) were negative. Anti Nuclear Antibody(ANA) profile was done and it was negative for autoimmune disorders such as Systemic Lupus Erythematosus(SLE), Sjogren's syndrome, Scleroderma, Polymyositis, Dermatomyositis, Systemic Sclerosis and Primary Biliary Cirrhosis. 


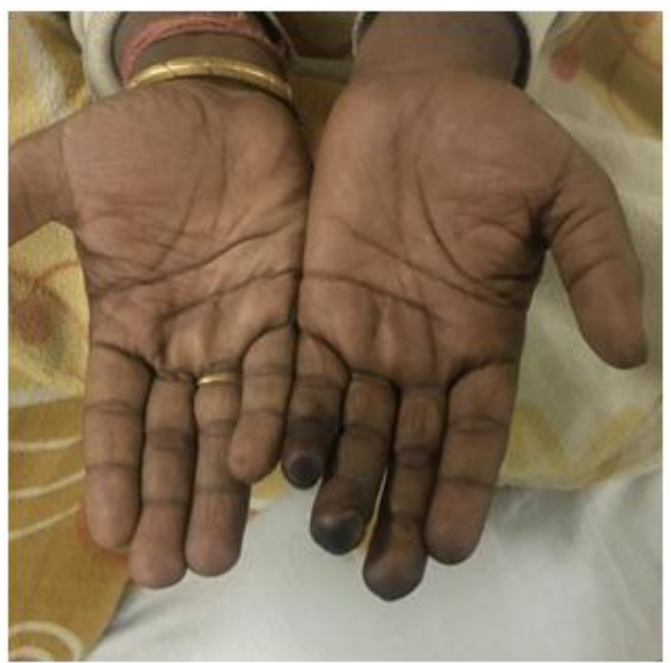

Fig 1. Bluish discolouration of 2 fingers of left hand with early involvementof the third finger

Left upper limb Doppler was done and showed narrowing of middle and distal portion of left brachial artery with collateral formation from proximal brachial artery. Left radial, ulnar and palmar arteries showed tardus et parvus waveform suggesting proximal obstruction. Right upper limb and bilateral lower limb Doppler were normal

The diagnosis of polyarteritis nodosa(PAN) and Granulomatosis with Polyangitis(GPA) was ruled out by the American College of Rheumatology criteria(ACR). Kawasaki disease was ruled out by the clinical criteria.

We diagnosed the patient with tuberculosis and secondary vasculitis. Patient was started on Anti Tubercular Therapy under Category II(treatment failure) as per RNTCP(Revised National Tuberculosis Control Program) guidelines. The symptoms drastically improved after starting therapy with isoniazide, rifampicin, pyrazinamide and ethambutol. A line of demarcation had formed and the symptoms of pain and swelling had decreased.

\section{Discussion}

Peripheral gangrene refers to situations that induce diminishing of the blood supply, nutrient, or oxygen to the tissues or organs for a prolonged period of time and lead to death of the tissues or organs. There are usually two types of gangrene - dry and wet. Dry gangrene is mainly a vascular gangrene. Malnourishment, diabetes mellitus, serious vascular impairment, and old age are predisposing factors.

A diagnosis of tuberculosis was based on history, favourable radiological and epidemiological features and suggestive laboratory investigations. Other causes of vessel obstruction and medium vessel vasculitis were ruled out. The patient improved once ATT was started.

The presence of gangrene in a case of tuberculosis has always been uncommon ${ }^{(3)}$. There is no clarity on the pathologic mechanism of such presentation.

There have been cases of peripheral symmetric gangrene in tuberculosis but these patients have had palpable peripheral pulses. Itin, et al. ${ }^{(4)}$ described symmetrical peripheral gangrene in a patient with disseminated tuberculosis. As mycobacterium tuberculosis was isolated from the blood culture, it was thought that symmetrical peripheral gangrene resulted from embolisation of arterioles by the tubercular bacilli. Hyperaggregation of platelets has also been thought to be a cause for gangrene with palpable peripheral pulses.

Vasculitis secondary to TB was first described in 1967 by Parish and Rhodes ${ }^{(5)}$. Tuberculosis is known to mimic large vessel vasculitis resembling Takayasu Arteritis but this commonly involves the aorta and its branches. Stratta et $\mathrm{al}^{(6)}$ suggested that M. tuberculosis was a chronic stimulus for an immunogenic reaction which could induce vasculitis process leading to vessel wall thickening, aneurysm formation, and stenosis of the vessel involved. Association of tuberculosis has also been noted with small vessel vasculitis, like leukoclastic vasculitis. To our knowledge, there has been only one case of medium vessel vasculitis caused by tuberculosis $^{(7)}$. The patient had vasculitis of the left posterior tibial artery and peroneal artery secondary to tuberculosis infection. Till now, there has been no reported case of TB involving vasculitis of mid and distal portion of brachial artery.

Gangrene secondary to tuberculosis has been reported at various sites of the body like scrotum, penis, small intestines and retina ${ }^{(8-10)}$ 


\section{Conclusion}

Due to the varied presentation of the disease, sometimes the diagnosis can be difficult. Peripheral gangrene and tuberculosis are rarely associated. But due to the widespread prevalence of disease, tuberculosis should always be excluded in a patient of gangrene, especially in TB endemic regions.

\section{References}

[1]. Global Tuberculosis Control 2015, WHO, Geneva, 2015 www.who.int/tb/publications/global_report/

[2]. DavisMD.Peripheralsymmetricalgangrene.MayoClinProc2004;79: 914.

[3]. Dubey AP, Sudha S, Parakh A. Peripheral gangrene: an uncommon manifestation of disseminated tuberculosis. Indian Pediatr 2006;43: 255-7.

[4]. Itin P, Stalder H, Vischer W. Symmetrical peripheral gangrene in disseminated tuberculosis. Dermatologica1986; 173: 189-195.

[5]. Parish WE, Rhodes EL. Bacterial antigens and aggregated gamma globulin in the lesions of nodular vasculitis. Br J Dermatol. 1967;79(3):131-47.

[6]. P. Stratta, A. Messuerotti, C. Canavese, M. Coen, L. Luccoli, B. Bussolati, et al. The role of metals in autoimmune vasculitis: epidemiological and pathogenic study. Sci Total Environ, 270 (2001), pp. 179-190

[7]. Yao Y, Liu B, Wang JB, Li H, Liang HD. Tuberculosis should not be ignored in patients with peripheral gangrene. J Vasc Surg 2010; 52:1662-4

[8]. Huriez C, Dupont A, Bergoend H, Thomas P, Douay B. Tuberculous gangrene of the penis. Bull Soc Fr Dermatol Syphiligr 1979: 91-92.

[9]. Derevianko IM. Gangrene of the scrotum of tubercular etiology. Urol Nefrol (Mosk) 1993; 5: 41-42.

[10]. Telkov NA. Gangrene of small intestines in tuberculosis of mesenteric glands. Probl Tuberk 1950; 4: 66-68. 